\title{
IMPROVING QoS IN DELAY TOLERANT MOBILE AD HOC NETWORK USING MULTIPLE MESSAGE FERRIES
}

\author{
R. C. Suganthe \\ Professor \\ Department of Computer Science and Engineering \\ Kongu Engineering College, Perundurai-638 052, Erode(dt),TamilNadu,India \\ Email: rc.suganthe@gmail.com_Phone:9842350051.

\section{P. Balasubramanie} \\ Professor \\ Department of Computer Science and Engineering \\ Kongu Engineering College, Perundurai-638 052, Erode(dt),TamilNadu,India
}

Received: November 21, 2011 Accepted: December 4, 2011 Published: December 31, 2011

DOI: 10.5296/npa.v3i4.1309 URL: http://dx.doi.org/10.5296/npa.v3i4.1309

\begin{abstract}
Most ad hoc network routing algorithms are designed primarily for networks that are always connected. While it is certainly desirable to maintain a connected network, various conditions may cause a mobile ad hoc network to become partitioned. If the partitions last for a long duration of time, then it is not possible to deliver a packet from source to destination. To deliver messages in a partitioned network, new routing approaches were proposed for delivering data using message ferries and/or gateways. This paper analyzes various parameters of the routing scheme called Routing with Multiple Message Ferries and Gateways (MMFGW) which eliminates online collaboration between all the communication entities in the network. This paper also investigates the results of simulations, by comparing the performance of our protocols which uses message ferries proposed previously with epidemic routing protocol. The simulation analysis shows that, delivery ratio of MMFGW
\end{abstract}


improves from $35 \%$ to $50 \%$ with optimal latency and overhead ratio.

Keywords: Delay Tolerant Networks, Delivery rate, Disconnected Ad hoc Network, Gateway, Latency, Message Ferry, Store-and-forward.

\section{Introduction}

In the rapidly growing age of IT, the sharing of vital information is necessary for many tasks and an urgent information can be disseminated the sooner or better a task can be completed. With the development of wireless technologies like GSM and Wi-Fi, information is often available anytime and anywhere. But the limitation of these technologies is that they require an infrastructure, i.e., base stations for their functioning. In environments such as disaster management or wartime conflict, this type of infrastructure is generally not available, but information exchange is still desired. An option to communicate in these environments is to use long range radios that enable point-to-point communication. The problems with these systems are that they are often expensive, bulky and provide only low bandwidth communication. Hence multihop wireless Ad hoc network is used. In a multihop wireless Ad hoc network, mobile nodes cooperate to form a network without using any infrastructure such as access points or base stations. Instead, the mobile nodes forward packets to each other, allowing communication among nodes outside the wireless transmission range.

Intermittently connected mobile ad hoc networks are mobile wireless networks where, for most of the time, a complete path does not exist from a source to a destination. Or such a path is highly unstable and may change or break soon after it has been discovered. This is due to constraints like node mobility, limited radio range, physical obstacles, inclement weather, wide deployment area or other physical factors [1]. Most ad hoc network routing algorithms are designed for networks that are always connected [2][3]. While it is certainly desirable to maintain a connected network, various conditions can cause a mobile ad hoc network to become partitioned. This means that there is no single-hop or multiple-hop route between some (or all) source/destination node pairs which might prevent some nodes from communicating with others and result in a partitioned network. The existence of network partitioning requires a new routing approach other than the traditional "store-and-forward" routing paradigm, used in most current ad hoc routing algorithms, in which messages are dropped if no route is found to reach a destination within a short period of time [4][5].

\subsection{Background}

The kind of communication networks addressed in this work are viable only for applications that can tolerate long delays and are able to deal with extended periods on being disconnected. In military war-time scenarios and disaster recovery situations, soldiers or rescue personnel are often placed in hostile environments where no infrastructure can be assumed to be present. Further, the units may be sparsely distributed and mobile so that connectivity between them is intermittent and infrequent [6]. In any large scale ad hoc 
network, intermittent connectivity is likely to be normal. Thus, meaningful research in this area is likely to payoff in practical systems.

\subsection{Delay Tolerant Network}

Delay-tolerant networks (DTNs) are designed to address networks that exhibit intermittent connectivity. Unlike conventional networks, DTNs do not necessarily provide end-to-end connectivity between two endpoints, and nodes may experience longer periods of disconnectivity than periods of connectivity. The latency to send a message may be of the order of hours or more, rather than fractions of a second. DTNs overcome the problems associated with intermittent, long or variable delay, asymmetric data rates and high error rates by using store and forward message switching[7].

DTN suggests combining all application level data and metadata to form a single bundled message called bundles, in order to minimize end-to-end transactions. The DTN architecture implement store-and-forward message switching by overlaying a new protocol layer called the Bundle Layer on top of heterogeneous, region-specific lower layers so that application programs can communicate across multiple regions. The bundle layer stores and forwards entire bundles or bundle fragments between nodes. A single bundle layer protocol is used across all networks (regions) that make a DTN. The layers which are defined in DTN are shown in Figure 1.

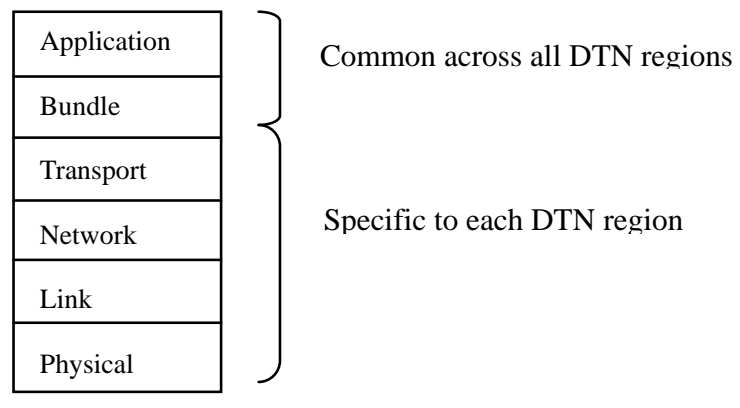

Figure 1. DTN layers

The most basic service provided by the bundle layer is its unacknowledged, prioritized unicast message delivery. It also provides two options for enhancing delivery reliability: end-to-end acknowledgments and custody transfer.

Since there may not be an end-to-end path in DTN networks, conventional end-to-end reliability mechanisms like retransmissions cannot work. DTN moves the responsibility of reliable delivery from a source node to other DTN nodes lying deeper in the network. This is achieved by moving a copy of message 'closer' to the destination. DTNs support node-to-node retransmission of lost or corrupted data at both the transport layer and the bundle layer. However, because no single transport layer protocol operates end-to-end across a DTN, end-to-end reliability can only be implemented at the bundle layer.

This paper is organized as follows: Section 2 describes the issues in traditional ad hoc 
routing protocols when frequent disconnections exist in the network. This section also discusses some of our protocols proposed for disconnected ad hoc network. In section 3 application scenario for intermittently connected network is described. We present a system model for QoS routing technique with multiple message ferries and gateways in section 4 . Section 5 dealt with the route length and connectivity of the network. Section 5 analyze the results of simulations, by comparing the performance of the proposed protocols with epidemic routing protocol. The performance is analyzed with varying node density, buffer space, mobility speed, transmission range, transmit speed and the number of messages. Section 6 illustrated the conclusion and future work.

\section{Related Work}

There are a number of routing protocols available for intermittently connected networks, where there is no guarantee that a fully connected path between source and destination exists at any time. It renders traditional routing protocols incapable of delivering messages between hosts. A number of routing schemes have been proposed to provide communications in highly partitioned networks[17]. Two of them are:

- Mobility assisted approach.

- Transmission power control protocol.

Several models, based on mobility assisted scheme, have been proposed to deal with routing in this type of network. The existing movement-assisted routing methods can be classified into two categories, based on the mobility control:

- $\quad$ Dissemination based approach or Random mobility model

- $\quad$ Message ferrying approach

Dissemination based approach uses the random mobility of nodes to transmit messages [8]. One of the best existing random movement schemes is epidemic routing(ER) $[9,10]$. Here, an assumption for this algorithm is that the nodes are all mobile and they have infinite buffers. It is a flooding-based algorithm. It means this: whenever a node has a message to send, it propagates the message to all nodes it meets and the nodes which receive the message continue to propagate it. Sooner or later, the data are delivered to the destination with a high probability. This approach can achieve high delivery ratios, and it operates without any knowledge of the network topology or communication pattern. It provides optimal delay only when the traffic is low. Yet, it is well-suited for networks where the contacts between nodes are unpredictable. Animal tracking networks such as SWIM and ZebraNet use random node mobility and flooding-based relaying.

Owing to the considerable number of transmissions involved, these techniques suffer from high contention and may potentially lead to network congestion[11]. To increase the network capacity, the spreading radius of a message is typically limited by imposing a maximum number of relay hops to each message, or even by limiting the total number of message copies present in the network at the same time.

In some situations, network partitions can last for a long period of time. For such network, dissemination based routing approaches are not suitable. Hence it cannot deliver 
messages between partitions which are mostly permanent or last for a long period of time.

In Transmission Power Control scheme, disconnected nodes in the network might be able to build their connection back to the network at increased transmission power [14]. When signal transmission power increases transmission range also increases but reduces the number of hops between mobile nodes. Less number of hops reduces the delay. On the other hand, by reducing signal transmission power, the probability of contention and collision in signal transmission is also reduced. Hence there is a trade off in controlling the signal transmission power in order to reduce an end-to-end message transmission delay.

Message Ferrying is, in fact, a controlled movement model, where nodes may change their original routes to collect and deliver messages [12]. Message Ferrying is a mobility-assisted proactive routing algorithm that incorporates message ferries(MF) by allowing communication among disconnected nodes. Ferries travel in a specified route, collecting data from sources and delivering data to the appropriate destinations. These message ferries allow nodes to communicate when the network is disconnected and when nodes do not have global knowledge of the network. It is a proactive routing algorithm created to address network partitions in intermittently connected ad hoc networks by establishing non-randomness in node movement. There are two types of nodes in MF scheme: Message ferries and regular nodes. This classification is based on their roles in communication. Ferries are mobile devices which take the responsibility of carrying messages among other nodes, while regular nodes are devices without any such responsibility. Several MF extensions could be carried out by installing multiple ferries[13] in a set of sub-regions through partitioning. This idea can be used in remote village communications and remote area connectivity projects for providing Internet access. MF scheme provides regular connectivity in a disconnected network and also improves the performance of data delivery without global knowledge of each node's location.

In our previous work, various routing approaches were proposed and analyzed for delivering data in disconnected mobile ad hoc network using message ferries: 'Controlled Epidemic Routing with Message Ferry(CMF) [18]', 'Routing with a Single Message Ferry and Gateways(SMFGW)' and 'Routing with Multiple Message Ferries(MMF) [19]'.

ER consumes more resources since the number of transmissions required per packet is more. So it is not scalable for huge network and also this approach is not suitable for message delivery between long-lived disconnected partitions. Message Ferrying approaches performed better for disconnected ad hoc networks. Routing with existing Message Ferrying scheme [12] needs online collaboration between Regular nodes and Message Ferry(MF), i.e., Message Ferry needs direct connectivity with a source and its destination. To remove online collaboration between regular nodes and Message Ferry, and to deliver packet between long-lived disconnected partitions, Epidemic routing and Message Ferry approaches were combined in CMF. This scheme scales well for huge disconnected network.

In CMF, if a message ferry does not visit any node in the destination cluster, then the message is not delivered to any node in the cluster during that visit. By using the stationary gateway in each cluster, a scheme SMFGW has been developed. This is aimed at improving the delivery rate and delay.

MMF improves the delivery rate with optimum latency and overhead. This scheme 
removes online collaboration between ferries and regular nodes but it needs online collaboration between ferries. The Routing with Multiple Message Ferries and Gateways (MMFGW) approach [20] eliminates online collaboration between all the communication entities in the network. This approach improves delivery rate with optimum latency and overhead. In general, the message ferrying scheme is the best solution for message delivery between network partitions which last for a long duration of time or stay permanently.

The approach MMFGW was proposed and only theoretically analyzed in [20]. All our approaches with message ferries are theoretically compared and analyzed in [21]. In this paper, we have simulated MMFGW in Opportunistic Network Environment (ONE) Simulator and analyzed for varying parameters like node density, mobility speed, delivery rate etc.

\section{Application Scenario}

This idea can be used in disaster relief missions, remote village communications and remote area connectivity projects for providing Internet access. Remote village communication means communication between disconnected villages. Ferries are special mobile nodes which have more resources than regular nodes. For example, buses [15] shuttle between remote villages which are equipped with memory (i.e. hard disks) and wireless interfaces can act as Ferries to collect and carry data among disconnected areas. One or more message ferry periodically visits each cluster/village to collect/deliver messages between disconnected nodes. The assumption here is that each MF follows a fixed and regular route and it makes a certain number of meeting points in its route. In remote village communication, a meeting point is an important place in the village where most of the people meet often regularly at the bus stand, market place etc. At this meeting point, the ferry has the longest contact time with the visited nodes for exchanging messages. In the regular/ Ferry nodes, messages will be dropped when the buffer overflows or the timeout expires. Timeout value depends on the delay requirement of the applications. Message ferrying is suitable for the application which can tolerate much delay, like file transfer, email and other non-real time applications.

\section{System Model}

The system analyzed in this paper uses multiple message ferries and gateways to make connectivity between nodes. Here two types of ferries are used: Global Message Ferry (GMF) and Local Message Ferry (LMF). For each cluster, there will be one LMF and one stationary Gateway node. Stationary Gateway nodes are deployed at important meeting points. While regular nodes can move anywhere in the deployed area, LMF may follow either a fixed route (called Multiple Message Ferries and Gateways with Fixed Route (MMFGWFR)) or a dynamically calculated route (called Multiple Message Ferries and Gateways with Dynamic Route (MMFGWDR)). 


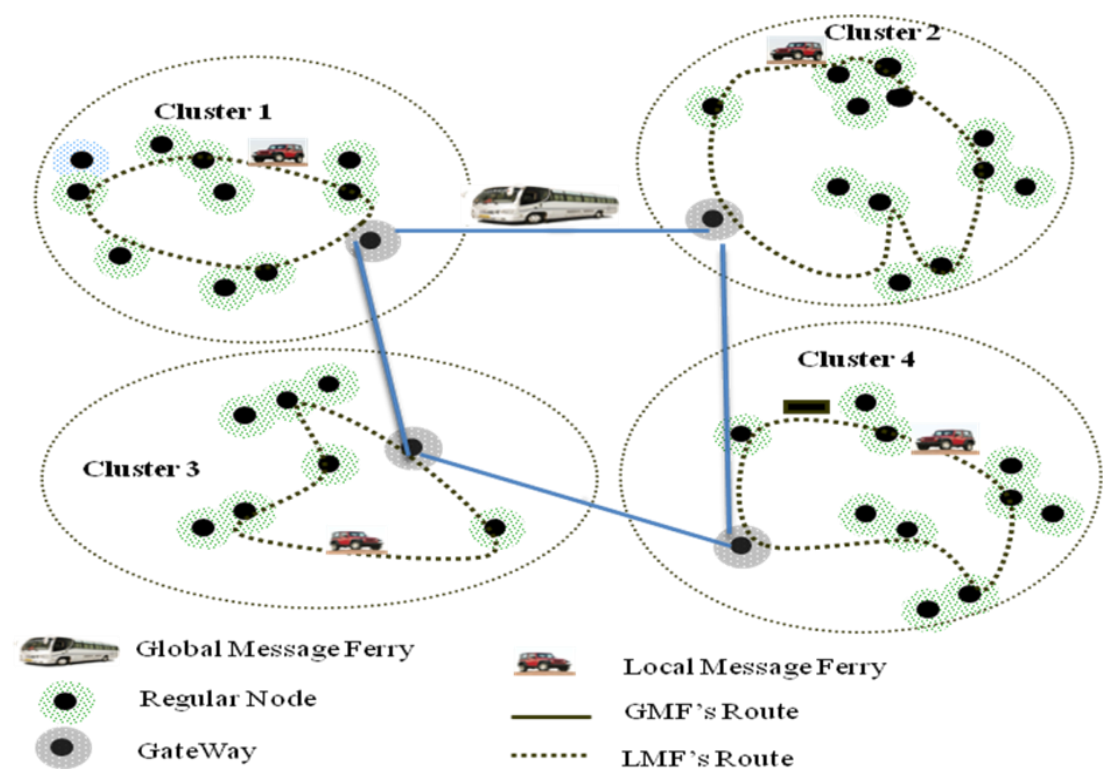

Figure 2. Routing with multiple message ferries and gateways

When LMF follows a fixed route, its route should be calculated carefully so that it covers a large number of nodes. The same route is used all the time. But if the LMF calculates the route dynamically, then the route is defined each time it starts its trip. This route is based on the position of nodes in the cluster. It is assumed that GMF follows a fixed regular route and makes a certain number of meeting points (where stationary gateway nodes are present) in its route. LMF receives/delivers messages from/to gateway/regular nodes. At this point, there is no need for online collaboration between GMF, LMF and also among regular nodes. GMF carries messages between clusters, whereas LMF carries messages between nodes within the cluster or exchanges message with Gateway. Whenever the source has a packet to send, it checks a route to its destination. If a route is found, then it delivers the packet. Otherwise, it delivers the packet to LMF. LMF periodically checks if there is any route to a destination for the packet stored in its buffer. If LMF finds the route, then it delivers the packet to the destination. If not, it exchanges the message with the Gateway. Whenever a connectivity occurs between GMF and Gateway, then they tend to exchange the undelivered packets. Figure 2 delineates Routing with Multiple Message Ferries and Gateways.

\subsection{Operation of Regular Nodes, Ferries and Gateways}

4.1.1 Node Operation: Operation of each regular node is explained as shown below as well as in the flow chart in figure 3 :

If a node has a packet to send, then perform the following:

1. If a route to a destination exists, then deliver the packet to its destination and remove this packet from its buffer.

2. If a route to LMF exists, then deliver the packet to LMF. Else, buffer the packet and wait for LMF's arrival. 


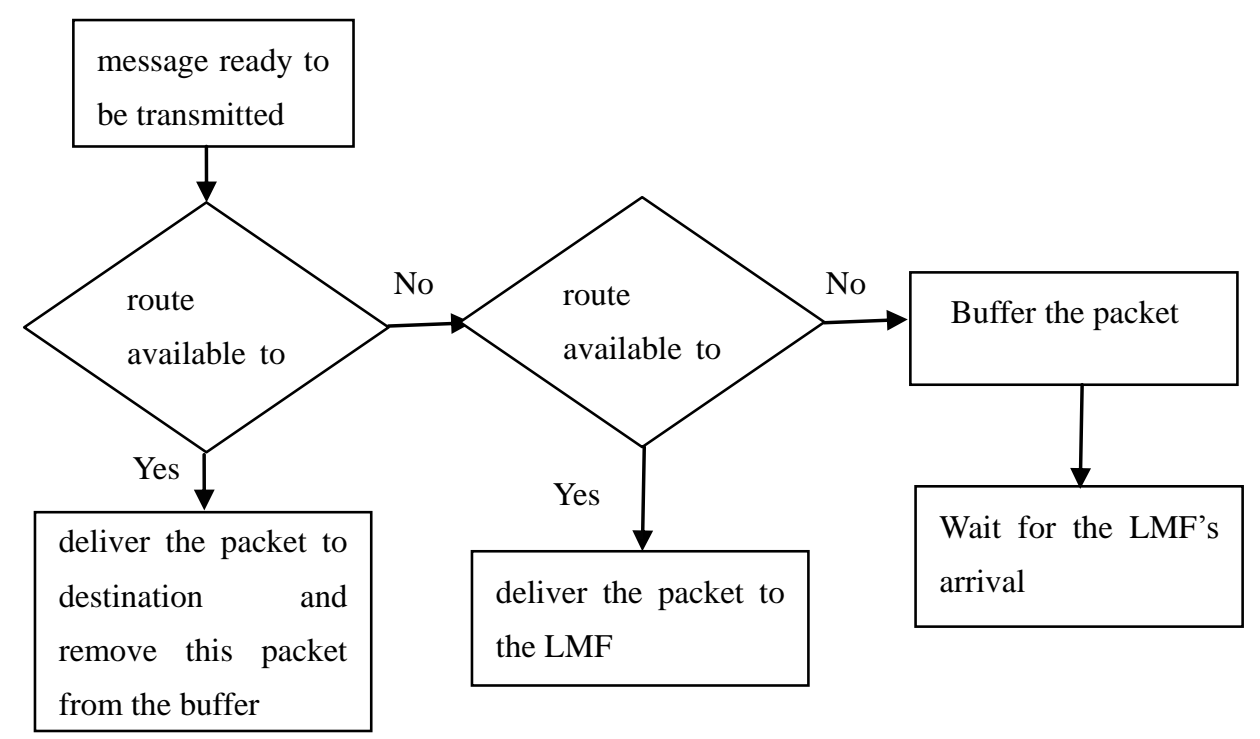

Figure 3. Node operation

4.1.2 Local Message Ferry's Operation: Local Message Ferry's operation is described (Figure 4) as shown below:

1. The LMF maintains a list of nodes which are located in its cluster, using GPS and it updates this information before calculating the route.

2. Before taking each trip, way points are selected for each partition in its cluster and the route is calculated so that it covers all the way points.

3. If a route exists for the buffered packet, then deliver the packet and remove it from its buffer. Else, wait for connectivity with the Gateway.

4. If LMF is within the range of Gateway, then exchange messages with the Gateway. 
Update location information for

all the nodes present in its

Select one node as way point for

each partition which contains

Calculate the shortest route

which connects all the way

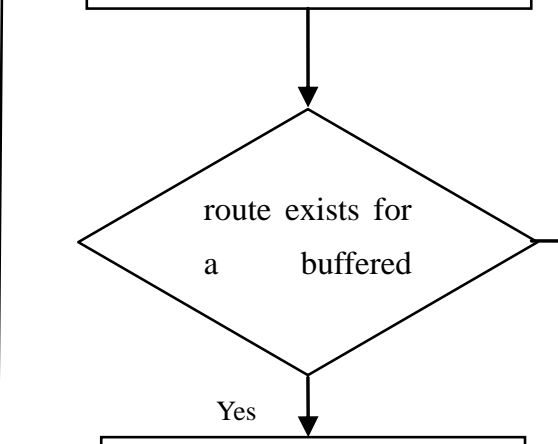

deliver the packet to

destination

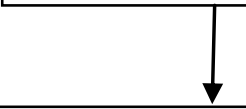

Remove delivered packet

from the buffer

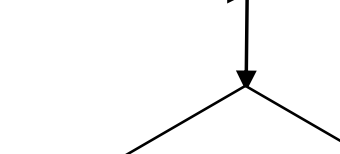

\section{Is $\mathrm{LMF}$ within}

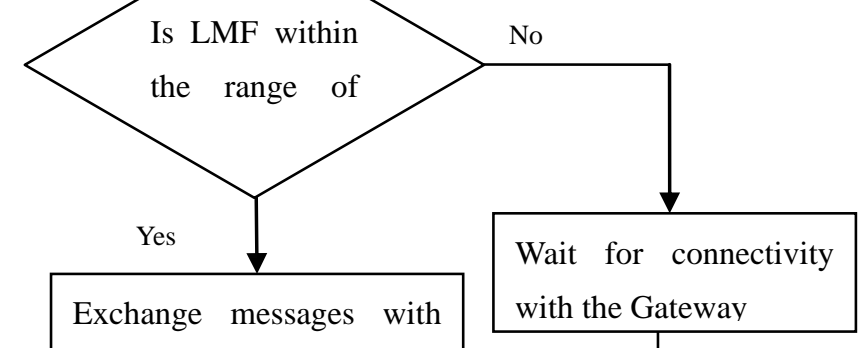
the Gateway

Figure 4. Local Message Ferry's Operation

4.1.3 Global Message Ferry's Operation: The GMF performs the following operation (Figure 5) if it has a buffered packet which is to be transmitted to the specific destination:

1. If a route exists for the buffered packet then

i. deliver the packet to its destination.

ii. remove the delivered packet from its buffer.

2. Else, if connectivity exists with the stationary Gateway and if a destination node is present in the stationary Gateway's cluster, then deliver the packet to the stationary Gateway. 


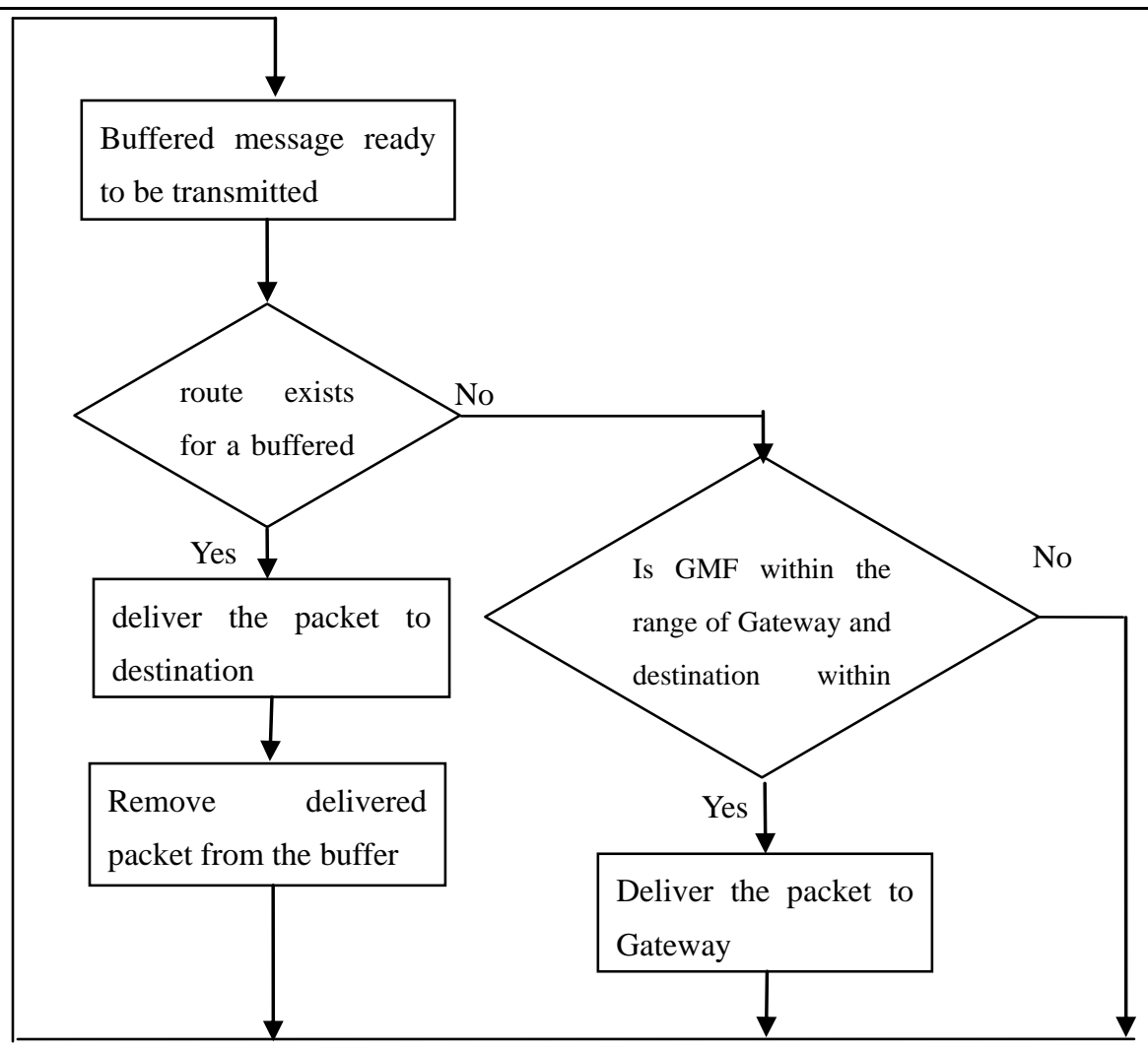

Figure 5. Global Message Ferry's Operation

4.1.4 Stationary Gateway's Operation: Figure 5 delineates the operation of Stationary Gateway. The Stationary Gateway performs the following sequence of operations if it has a buffered packet for transmission:

1. If a route exists for the buffered packet then,

a. deliver the packet to its destination.

b. remove the delivered packet from its buffer.

2. Else, if connectivity exists with the LMF and if a destination node is present in its cluster, then,

a. deliver the packet to LMF.

b. remove the packet from its buffer.

3. Else, if connectivity exists with the GMF, then, exchange messages with the GMF. 


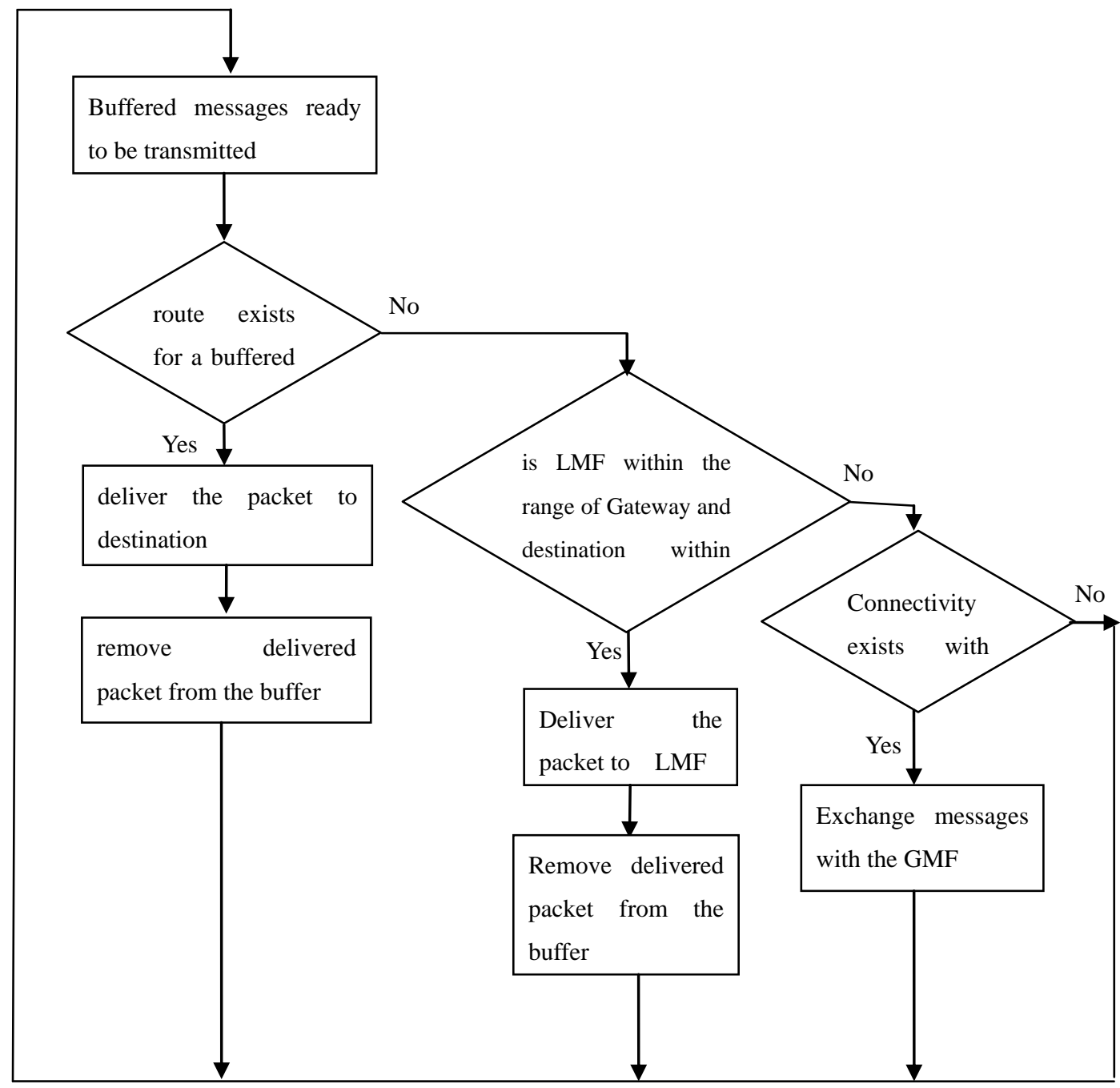

Figure 6. Stationary Gateway's Operation

\subsection{Algorithm for dynamic route calculation of LMF}

\subsubsection{Find the number of neighbors for each node in the cluster}

The following algorithm finds number of neighbors for each node in the cluster based on connectivity with other nodes in the cluster. Number of neighbor for a particular node is equal to number of nodes in its transmission range.

a. Let A be the set of ' $q$ ' nodes within the cluster,

$$
\mathrm{A}=\left\{n_{1}, \ldots, n_{q}\right\}
$$

b. For each node $n_{i}$ in A,

$$
\text { Begin }
$$

Initialize the number of neighbors of $n_{i}$ to 0 
i.e., no-of-neighbor $\left(n_{i}\right)=0$

For each node $n_{j} \neq n_{i}$ in $\mathrm{A}$

Begin

If $n_{j}$ is neighbor $\left(n_{i}\right)$ then

$$
\text { no-of-neighbor }\left(n_{i}\right)=\text { no-of-neighbor }\left(n_{i}\right)+1 \text {; }
$$

End

End

\subsubsection{Find way points for route calculation}

This algorithm selects a node which has the highest neighbor as way point in each partition. The partition is defined as nodes in one partition cannot communicate with nodes in other partition. The way point for each partition is selected using the following algorithm:

a. Mark all the nodes in A as "unvisited"

b. Find the highest neighbor node for each partition in the cluster.

Let $\mathrm{B}=\{\phi \quad\}$ be the set of highest neighbor nodes, called "way point list".

While (any unvisited node exists in A) do

begin

heighest-neighbor $=0$

for each unvisited node $n_{i}$ in A do

begin

if no-of-neighbor $\left(n_{i}\right)>=$ highest-neighbor then

$$
\begin{aligned}
& \text { highest-neighbor }=\text { no-of-neighbor }\left(n_{i}\right) \\
& \text { highest-neighbor-node }=n_{i}
\end{aligned}
$$

end

$\mathrm{B}=\mathrm{B} \cup$ highest-neighbor-node

Mark highest-neighbor-node and all the nodes reachable from this node as "visited".

end

4.2.3 Design a route which covers the location of all the nodes in set B using the Shortest Path algorithm (TSP algorithm).

The following shortest path algorithm finds the shortest path from Message Ferry 
location $(x, y, z)$ to all the way points in the way points list ' $\mathrm{B}$ ':

function ShortestPath (Way Point List, Message Ferry location):

for each node $\mathrm{v}$ in Way Point List: // Initializations

$\operatorname{dist}[\mathrm{v}]:=$ infinity // Unknown distance from source to $\mathrm{v}$

previous $[\mathrm{v}]:=$ undefined // Previous node in optimal path from source

dist[Message Ferry] $:=0 \quad$ // Distance from source to source is 0

$\mathrm{Q}:=$ the set of all nodes in Way Point List // All nodes placed in Q

while $Q$ is not empty

$\mathrm{u}:=$ node in $\mathrm{Q}$ with smallest distance with the message Ferry

remove $u$ from $Q$

for each neighbor $v$ of $u$ : // where $v$ has not yet been removed from $Q$.

temp := dist $[\mathrm{u}]+$ dist_between $(\mathrm{u}, \mathrm{v})$

if temp $<\operatorname{dist}[\mathrm{v}]$

$\operatorname{dist}[\mathrm{v}]:=$ temp

previous $[\mathrm{v}]:=\mathrm{u}$

return previous[]

\section{Route length and cluster connectivity}

Let ' $n$ ' be the number of clusters in the network. For each cluster, there is one stationary Gateway and one LMF. Let $G=\left\{G_{1}, \ldots, G_{n}\right\}$ be the set of Gateways and $L=\left\{L_{1}, \ldots, L_{n}\right\}$ be the set of LMFs. Let $d_{i j}$ be the distance between Gateway $G_{i}$ and $G_{j}$ in meters. Global Ferry visits all the Gateways in the network. Hence, the route length of the Global Ferry is the sum of the distance between all the Gateways in its route i.e., Route Length of the Global Ferry = $\sum \mathrm{d}_{\mathrm{ij}}$ which is calculated using Equation 1. Assume the speed of the Global Ferry as X meters/seconds and Local Ferry as Y meters/seconds. The waiting time for GMF at each Gateway $G_{i}$ is $W_{i}$, where $i=1,2, \ldots, n$. The time taken to complete each round of GMF is calculated using equation 2. If the waiting time at each gateway is the same (W seconds), then the time taken to complete each round of GMF is calculated using equation 3. Once in the duration of $t$, the Global Ferry creates regular connectivity between clusters.

$$
\begin{aligned}
& \sum \mathrm{d}_{\mathrm{ij}}=\mathrm{d}_{12}+\mathrm{d}_{23}+\mathrm{d}_{34} \ldots \ldots+\mathrm{d}_{(\mathrm{n}-1) \mathrm{n}}+\mathrm{d}_{\mathrm{n} 1} \\
t= & \frac{\sum d_{i j}}{X}+\sum_{k=1}^{n} W_{k}
\end{aligned}
$$

(Equation 2) 


$$
t=\frac{\sum d_{i j}}{X}+(n * W)
$$

(Equation 3)

If delivery rate alone is considered, then there is no restriction on the arrival time of LMF $L_{j}$ and GMF. But when considering delivery delay, this depends to some extent, on the arrival time of LMF $L_{j}$ and GMF.

Let the arrival time of GMF be, $T g_{j}$ and LMF $L_{j}$ is $T l_{j}$ at the Gateway $G_{j}$. There are a number of conditions for analyzing message delay with respect to arrival time:

If $a b s\left(T g_{j}-T l_{j}\right)<\left(W_{j} / 2\right)$ then, the messages from LMF and GMF are exchanged almost immediately. In this condition, half of the waiting time is used for exchanging the messages.

If $T g_{j}+W_{j}=T l_{j}$, then, the $L_{j}$ immediately receives the message from the gateway, without any wastage of buffering time for the message at the gateway. But buffered messages, destined to other cluster in LMF, cannot be delivered to the GMF. Hence, the delivery delay for the message destined to the current cluster is less, while the message destined to other cluster from the current cluster is high.

If $\left(T g_{j}>T l_{j}+W_{j}\right)$ is true, then, LMF leaves the Gateway before the GMF arrives at the gateway. If $\left(\left(T g_{j}-\left(T l_{j}+W_{j}\right)\right) * Y\right)$ is very less, then, the LMF has to travel a longer distance to reach the Gateway. In this situation, the LMF takes more time to the reach Gateway. Hence the average delay due to buffering at the gateway will be high. If $\left(\left(T g_{j}-\left(T l_{j}+W_{j}\right)\right) * Y\right)$ is more, then, the LMF has to travel only a shorter distance to reach the Gateway. So, the LMF takes less time to reach the gateway after the message is delivered by the GMF. In this situation, buffering time is high though the delay will be longer.

If $\left(T g_{j}+W_{j}<T l_{j}\right)$, then, GMF leaves before the LMF arrives at the gateway. If $\left(\left(T l_{j}-\left(T g_{j}+W_{j}\right)\right)^{*} Y\right)$ is less than half of the route length of the LMF, then the average delay will be less since LMF takes less time to reach the gateway after the message is delivered by the GMF. If $\left(\left(T l_{j}-\left(T g_{j}+W_{j}\right)\right) * Y\right)$ is high, then, LMF takes more time to reach the gateway. So the delay will be high. However, the route length of LMF varies depending on the location of the nodes. 


\section{Result Analysis}

Simulator used for this work is Opportunistic Network Environment (ONE) simulator. The simulation period for each scenario is 20,000 seconds and the simulated mobility network area is $5400 \mathrm{~m} \times 5500 \mathrm{~m}$ rectangle. Similarly, the network area is divided into three disconnected clusters. Nodes can move anywhere in the cluster. This section analyses the results of simulations, by comparing the performance of the proposed protocols with epidemic routing protocol. Next, the performance is analyzed with varying node density, buffer space, mobility speed, transmission range, transmit speed and the number of messages. Performance analysis of MMFGWDR and MMFGWFR over ER are illustrated in Figures 7 to 14 .

Number of nodes vs. Delivery rate: Now assume that the buffer size is unconstrained. Message Ferry achieves an improvement in performance that is better than that of epidemic routing protocol. Epidemic routing suffers from the inability to deliver messages to recipients that are in other disconnected cluster. In this protocol, message is propagated only to the accessible hosts until the TTL of the message expires. When TTL of the message expires, the message will be dropped. One reason for message dropping is that the recipient remains in the same disconnected cluster for a long duration of time which is longer than that of TTL of the message. In the MMFGW scheme, the message is carried by GMF and creates regular connectivity between clusters. Message delivery within each cluster is performed by LMF and Gateway. LMF creates connectivity between almost all the nodes in the cluster. This scheme does not require any online collaboration between regular nodes, GMF and LMF. But this will increase the probability of delivering the packet. The simulation results in figure 7 show that delivery rate improves from $30 \%$ to $45 \%$ in MMFGWFR and $35 \%$ to $50 \%$ in MMFGWDR for varying number of nodes compared to ER with optimal end-to-end delay and overhead ratio.

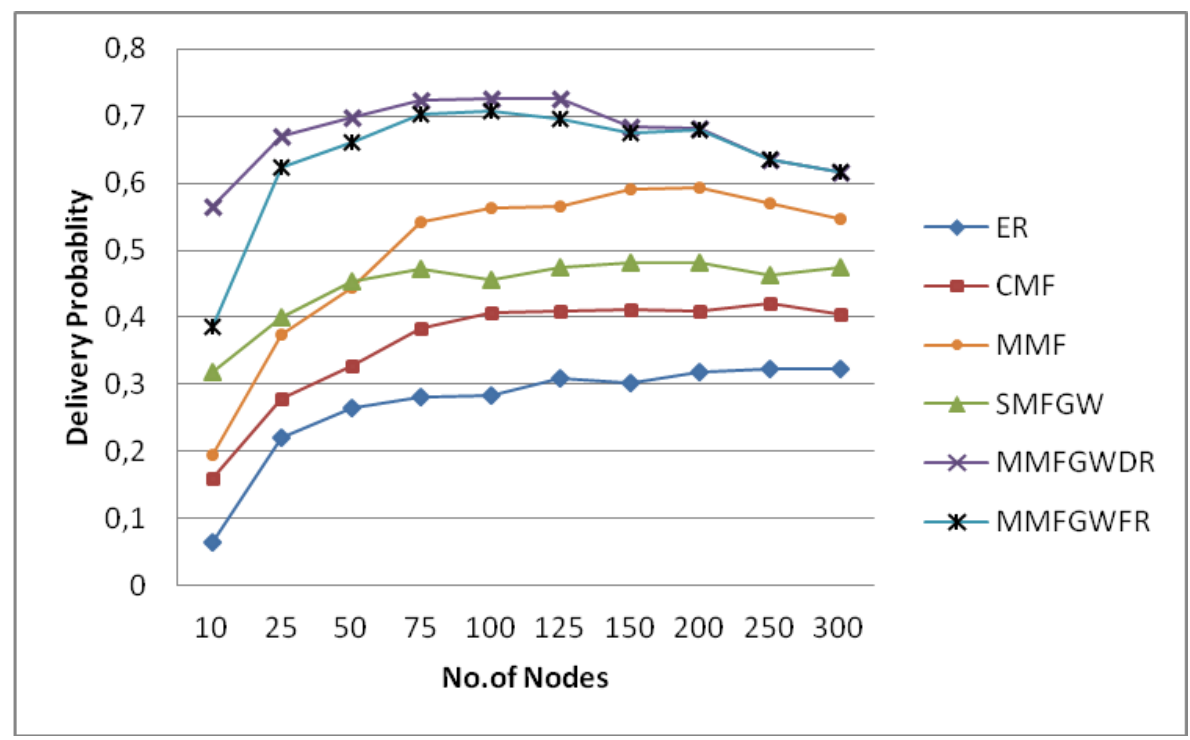

Figure 7. Number of nodes vs Delivery probability 
Mobility speed vs. Delivery probability: As shown in figure 8 upto certain limit, delivery probability increases if mobility speed increases. This is due to mobility creates connectivity between nodes. If speed increases beyond the limit, nodes can not have a time to exchange the messages. Hence delivery probability will be reduced.

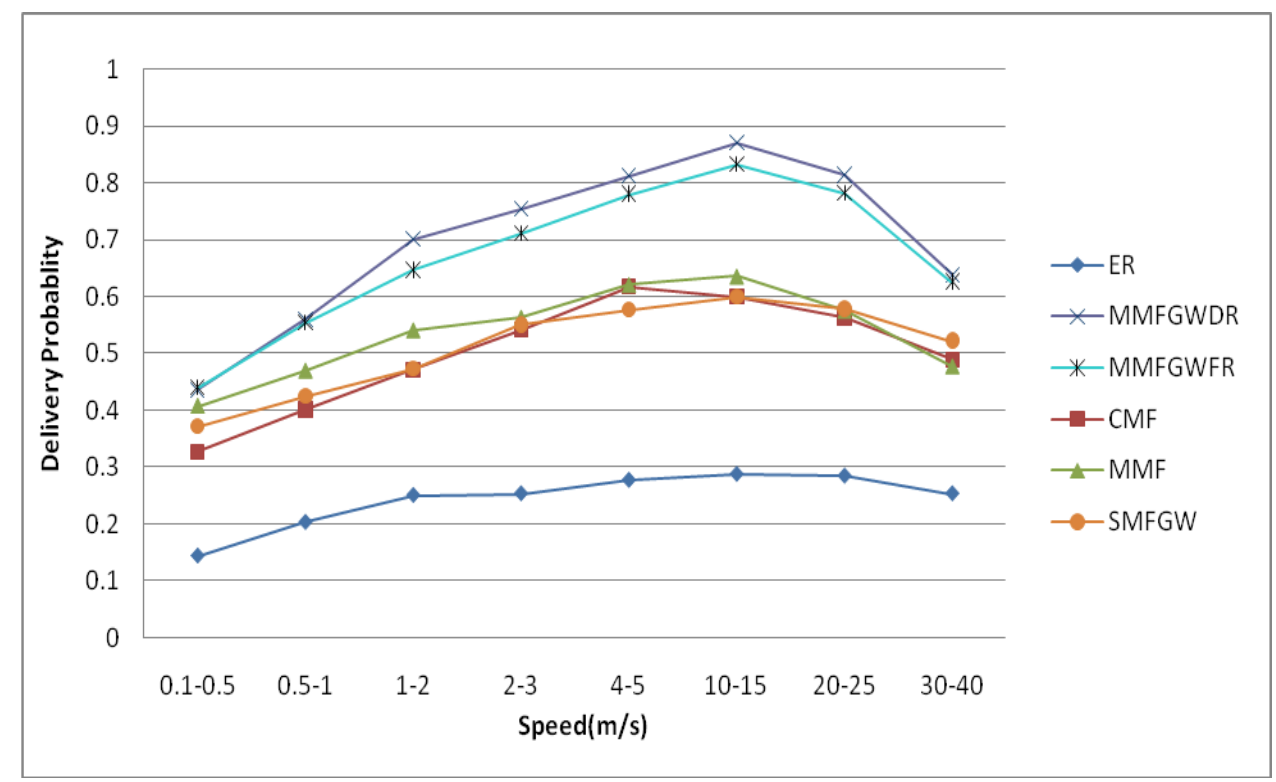

Figure 8. Mobility speed vs Delivery probability

Number of nodes vs. Delivery delay: In epidemic routing protocol, the number of nodes increases the connectivity between nodes and thus reduces the delay. This improvement is only upto a certain limit because more number of nodes increases the congestion. If the destination is in the same cluster as the source or if a route exists between a source and its destination then the message is delivered more or less immediately in both the protocols. Consider the situation in which the destination is in other cluster which is disconnected from the source cluster. In this situation, whenever connectivity occurs due to mobility of the node before the lifetime of the packet expires is only delivered in epidemic routing protocol. If delivery is more important than any other metric, the node has to wait for connectivity. This increases delay time. But in the MMFGW scheme, Global Ferry makes connectivity among clusters periodically and also Local Ferry makes connectivity among disconnected nodes within the cluster. This will reduce delivery delay (Figure 9). If the node has connectivity with the Gateway, then, the message is delivered quickly. If the message is far away from the Gateway, then the message is delivered to LMF and LMF delivers it to the destination, whenever a route to a destination occurs. 


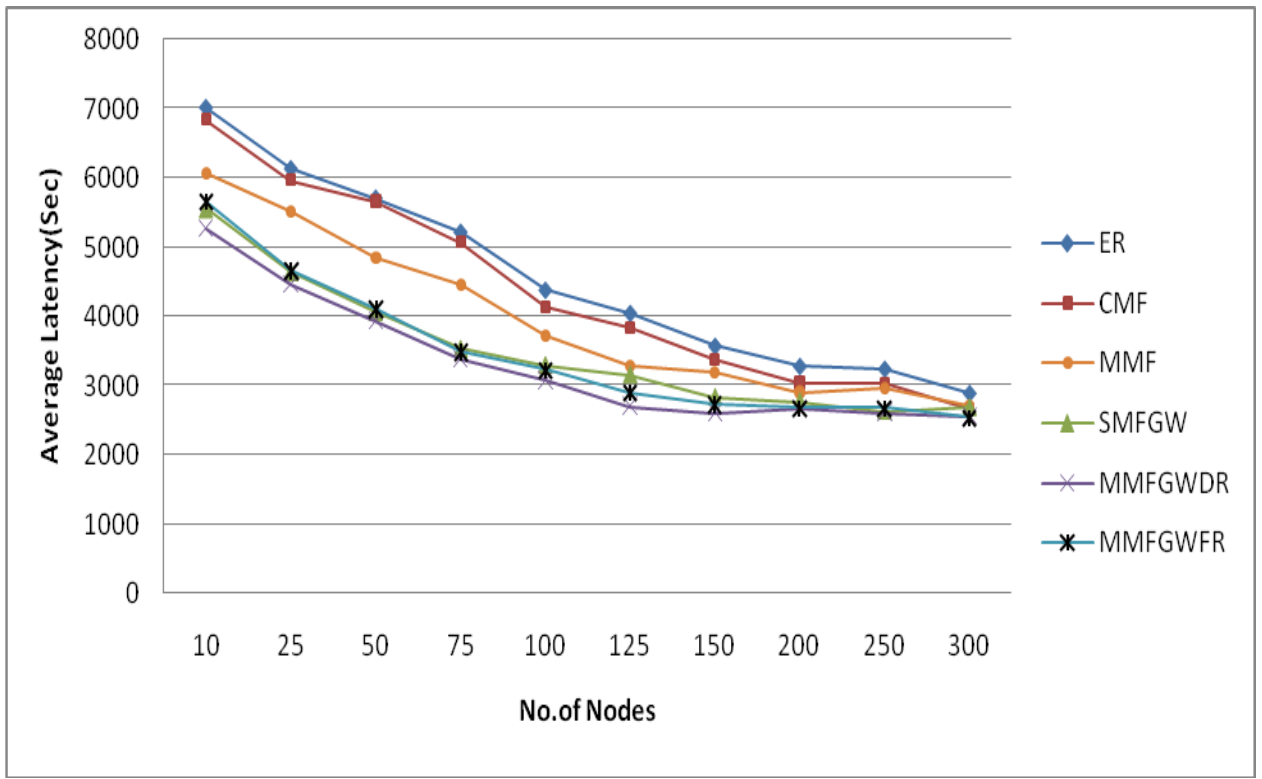

Figure 9. Number of nodes vs Average latency

Transmission range vs. Delivery probability: The figure 10 shows that, upto certain limit, delivery probability increases if transmission range increases. This is due to increase in transmission range creates connectivity between nodes. If transmission range increases beyond certain limit, probability of contention and collision in signal transmission is also increases. Hence delivery probability will be reduced.

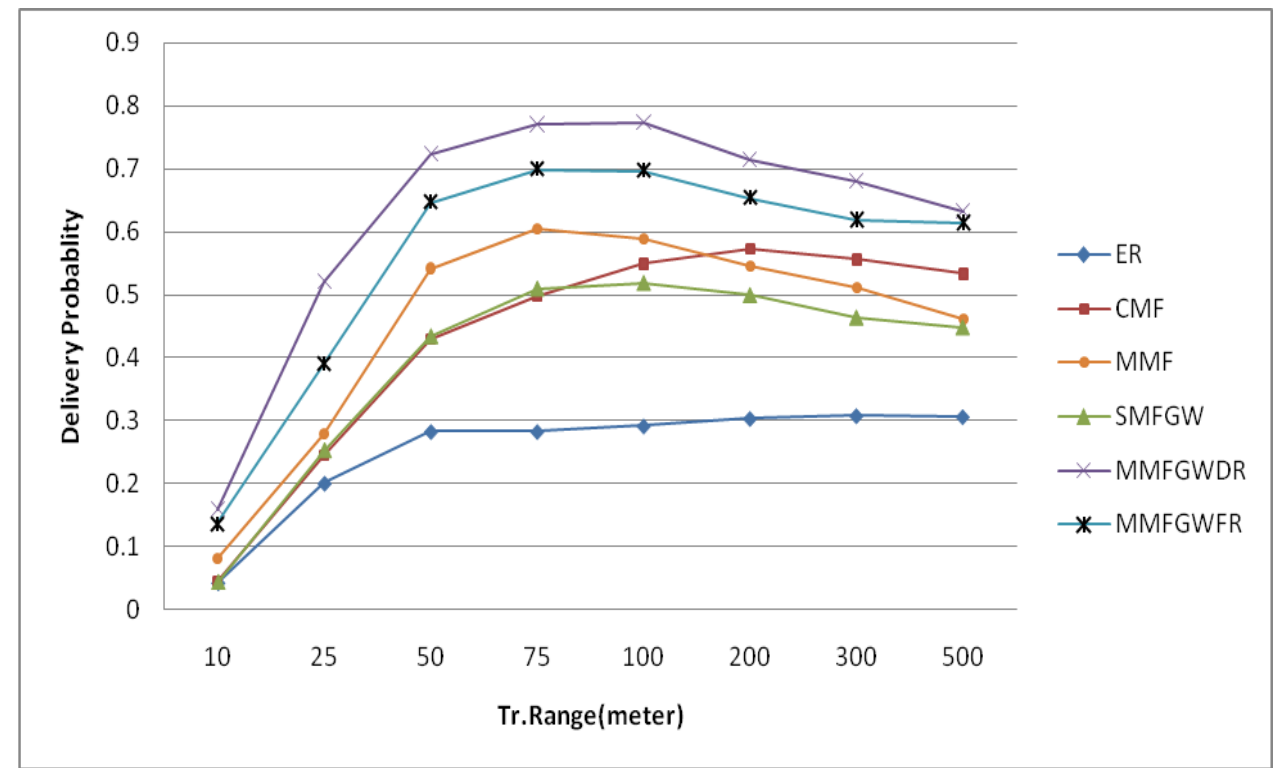

Figure 10. Transmission range vs Delivery probability

Buffer size vs. Delivery probability: When the buffer size is small, the probability of message dropping will be high and the number of messages exchanged also will be low. At the other end, as buffer size increases, the number of message drop will be reduced due to an overflow. This will improve delivery probability as shown in figure 11. In general, as the 


\section{Al Macrothink}

buffer space increases, the data delivery ratio also increases. On the other hand, with a limited buffer space, new packets may replace the old undelivered ones. This results in packet drops and a low delivery ratio. Epidemic routing protocol propagates the packet to all the accessible hosts. All the hosts in the network are required to exchange message for all the remaining nodes in the network. Hence all the nodes need more buffer space. If the number of nodes is increased then nodes need to have more buffer space. In the MMFGW protocol, regular nodes deliver messages to destination/LMF and also receive messages which are intended for it. Hence regular nodes not require more buffer space and buffer space of regular nodes does not affect delivery rate. Gateway and LMF also not require huge buffer space because they carry messages for the nodes which are located in their cluster. GMF carries messages among clusters hence it needs more buffer space than LMF. Hence nodes in the new scheme may require only a very small amount of buffer space than that of epidemic routing protocol.

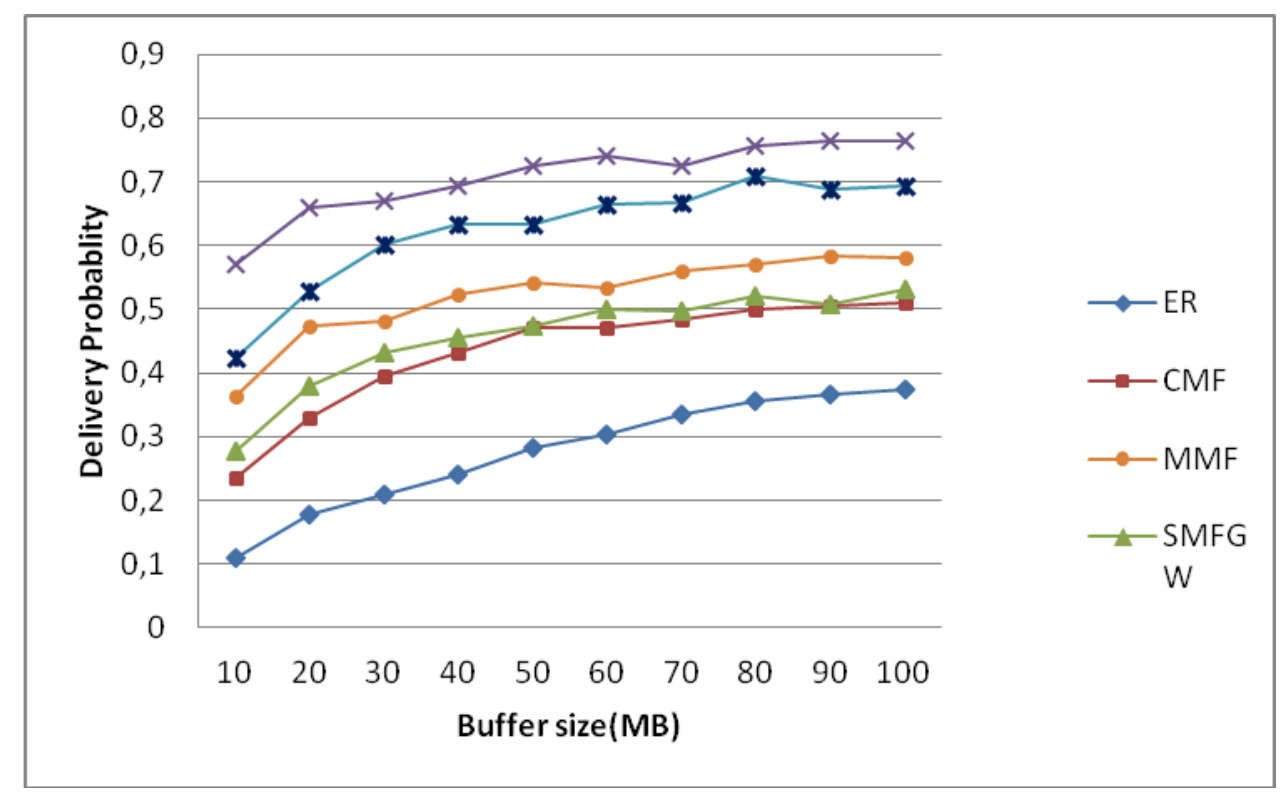

Figure 11. Buffer size vs Delivery probability

Number of Messages vs. Delivery probability: When the buffer size is large enough to hold all the messages delivery probability is high and it is based on other factor such as connectivity, mobility speed etc. When number of messages increased beyond the capacity of the buffer, messages which can not accommodate the buffer will be dropped. Hence delivery probability will be reduced. The result of our simulation is shown in figure 12 . 


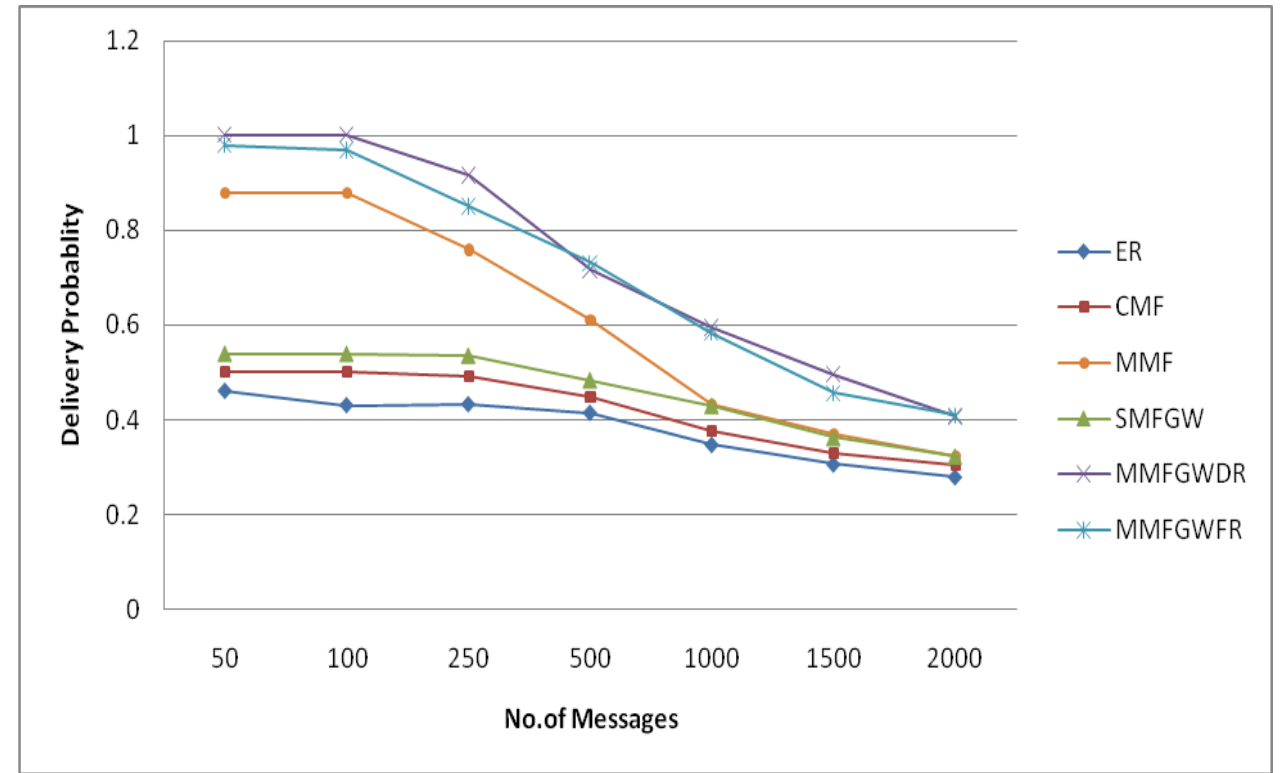

Figure 12. Number of messages vs Delivery probability

Number of nodes vs. Overhead ratio: When number of nodes increases overhead ratio also increases since number of transmissions per message increases. The simulation result is shown in figure 13 ,

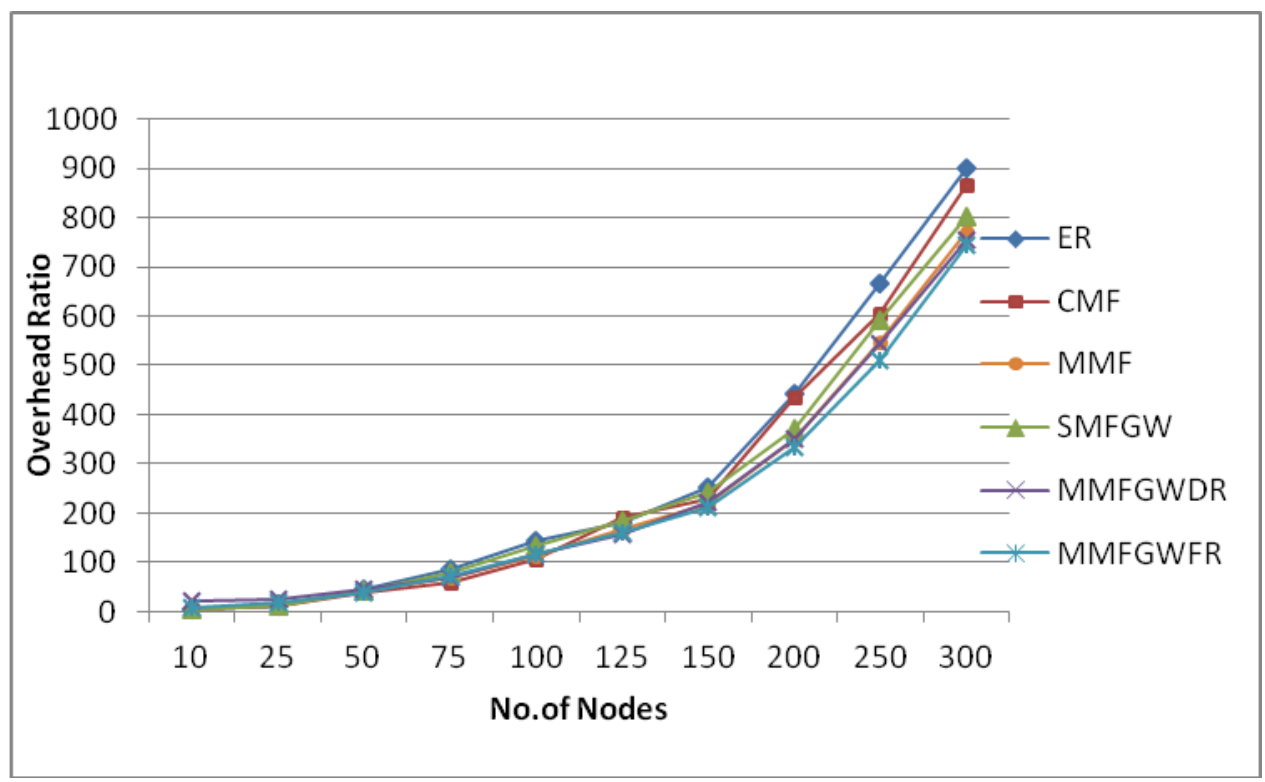

Figure 13. Number of nodes vs Overhead ratio

Resource Utilization: In Epidemic routing protocol, all the nodes in the network store and carry the message. Hence all the nodes need more resources. In the new improved scheme, regular nodes not carry the messages and thus need less resource. But GMF, LMF and Gateway nodes need to buffer the messages and thus need more resources. 
Computational overhead: Epidemic routing protocol needs less computational power since there are no special nodes in the network. Also in this case, nodes just store, carry and forward the message. For providing a better performance, all the nodes in the network should implement a buffer allocation policy. The new proposed schemes use a number of special nodes called GMF, LMF and Gateway. Regular nodes do not provide any special function other than forwarding the message, if a route exists to destination. Hence regular nodes do not require any additional computation. GMF follows a fixed regular route and carries the message between disconnected partitions. So it just implements a buffer allocation policy for a better performance and it does not require additional computational power for calculating the route. LMF, on the other hand, dynamically calculates the route, based on the current location of the nodes before starting each trip and also it carries the message to regular nodes. Thus, it needs more computational power than GMF. Gateway nodes just buffer and deliver the message and they do not require additional computational power.

\section{Conclusion and future work}

In this paper, a novel routing scheme using Message Ferry and Gateway is proposed for delay tolerant mobile ad hoc networks. The analysis shows that, MMFGWFR has $30 \%$ to 45\% while MMFGWDR has $35 \%$ to $50 \%$ higher delivery ratio with optimal end-to-end delay, average buffer time, hop-count average and overhead ratio compared to ER for varying number of nodes from 10 to 300. Certainly, MMFGWFR improves delivery rate from $30 \%$ to $54 \%$ and MMFGWDR from $30 \%$ to $58 \%$, that is more than ER for varying mobility speed of the nodes. Further analysis indicates that MMFGW's relative performance is better than ER in terms of delivery rate, average end-to-end latency and overhead ratio for varying transmit speed, transmit range, number of messages and buffer space. This scheme removes online collaboration between all the communication entities and this is certainly an advantage over other systems discussed in the preceding chapters.

The simulation results show that CMF and SMFGW produce good delivery ratio only when connectivity exists between nodes within the cluster. If partition occurs between nodes in the cluster, then, routing schemes with multiple ferries like MMF, MMFGWFR and MMFGWDR produce a higher delivery ratio than other techniques.

Future researchers would do well by making investigations into the impact of different classes of traffic with efficient buffer allocation schemes in so far as Ferries and Gateways are concerned in the ever expanding field of mobile ad hoc networks. In the future research, more analytical study with network throughput analysis can be included. Multicasting in Delay tolerant mobile ad hoc network can also be investigated in the future.

\section{References}

[1] Li Q. and Rus D., Communication in disconnected ad hoc networks using message relay, 
Journal of Parallel and Distributed Computing, vol. 63 No.1 (2003) 75-86. http://dx.doi.org/10.1016/S0743-7315(02)00033-3

[2] Perkins C. and Belding-Royer E, Ad hoc on-demand distance vector (aodv) routing, Rfc 3561 (2003). www.ietf.org/rfc/rfc3561.txt

[3] Zhou H ,A Survey on Routing Protocols in MANETS, MSU- CSE-03-08 (2003).

[4] Abolhasan M., Wysocki T. and Dutkiewicz E, A review of routing protocols for mobile ad hoc networks, Ad Hoc Networks Elsevier Vol.2 No.1 (2004), Pp. 1-22. http://dx.doi.org/10.1016/S1570-8705(03)00043-X

[5] Mundur P., Lee S. and Seligman M, Routing for Data Delivery in Dynamic Networks, Military Communications Conference MILCOM 2006, IEEE Washington, DC, 1-7. http://dx.doi.org/10.1109/MILCOM.2006.302555

[6] Small T. and Haas Z. J., Resource and performance tradeoffs in delay-tolerant wireless networks, proc. of the 2005 ACM SIGCOMM workshop on Delay-Tolerant Networking, ACM New York, NY, USA, 260-267. http://dx.doi.org/10.1145/1080139.1080144

[7] Cerf V., Burleigh S., Hooke A., Torgerson L., Durst R., Scott K., Fall K. and Weiss H., Delay-Tolerant Networking Architecture, RFC 4838(2007). http://www.ietf.org/rfc/rfc4838.txt

[8] Grossglauser M. and Tse D.N.C., Mobility increases the capacity of ad hoc wireless networks, IEEE/ACM Transactions on Networking Vol.10 No.4 (2002) 477 486. http://dx.doi.org/10.1109/TNET.2002.801403.

[9] Vahdat A. and Becker D, Epidemic routing for partially connected ad hoc networks, Technical Report CS-200006, Duke University. (2000). http://issg.cs.duke.edu/epidemic/epidemic.pdf

[10] Khelil A., Becker C., Tian J. and Rothermel K., An Epidemic Model for Information Diffusion in MANETs, Proc. of the 5th ACM international workshop on Modeling analysis and simulation of wireless and mobile systems MSWiM, ACM New York, NY, USA 2002, 54 - 60. http://dx.doi.org/10.1145/570758.570768

[11] Zhang X., Neglia G., Kurose J. and Towsley D., Performance modeling of epidemic routing, International Journal of Computer and Telecommunications Networking, Vol.51 No.10 (2007) 2867-2891. http://dx.doi.org/10.1016/j.comnet.2006.11.028

[12] Zhao W., Ammar M., and Zegura E, A message ferrying approach for data delivery in sparse mobile adhoc networks, In Proc. of fifth ACM International Symposium on Mobile Ad Hoc Networking and Computing, May 24-26, 2004, MobiHoc 2004, Tokyo, Japan, 2004. http://dx.doi.org/10.1145/989459.989483

[13] Zhao W., Ammar M., and Zegura E., Controlling the mobility of multiple data transport ferries in a delay-tolerant network, Proc. of 24th Annual Joint Conference of the IEEE Computer and Communications Societies, 13-17 March 2005, Miami, FL, USA. IEEE INFOCOM 2005, 1407-1418. http://dx.doi.org/ 10.1109/INFCOM.2005. 1498365

[14] Kawadia V and Kumar P.R., Principles and protocols for power control in wireless ad hoc networks' IEEE Journal on Selected Areas in Communications, Vol. 23 No. 1, (2005) 76-88. http://dx.doi.org/10.1109/JSAC.2004.837354\%28410\%29\%2023

[15] Nekovee M., Bogason B.B 'Reliable and Effcient Information Dissemination in Intermittently Connected Vehicular Adhoc Networks' Vehicular Technology Conference, 


\section{Macrothink}

Dublin, Ireland,VTC2007-Spring. IEEE Vol.65 No.22-25 pp. 2486 - 2490. 22 - 25 April 2007, http://dx.doi.org/10.1109/VETECS.2007.512

[16] Delay Tolerant Networking Research Group, Internet Research Task Force, October (2002) http://www.dtnrg.org/

[17] Suganthe R.C and Balasubramanie P. Routing in Intermittently connected Mobile Ad hoc Networks: A Survey, International Journal of Engg. Research and Industrial Applications, Vol. I No.6, (2008) 223-244.

[18] Suganthe R.C and Balasubramanie P. Improving Delivery Rate In Intermittently Connected Mobile Ad Hoc Network, International Journal of Engineering Research and Industrial Applications, Vol.I No.7 (2008) 309-322.

[19] Suganthe R.C and Balasubramanie P. Improving Qos In Disconnected Mobile Ad Hoc Network, Journal of Mobile Communications Vol. 2 No.4 (2008) 99-104. http://medwelljournals.com/abstract/?doi=jmcomm.2008.99.104

[20] Suganthe R.C and Balasubramanie P. Efficient Routing for Intermittently Connected Mobile Ad-Hoc Network, International Journal of Computer Science and Network Security, Vol. 8, No. 11, (2008) 184-191.

[21] Suganthe R.C and Balasubramanie P. Comparison of QoS Routing Approaches for Intermittently Connected Mobile Ad-Hoc Network, International Journal of Applied Engineering and Research, Vol. 4, No. 2 (2009) 175-185.

\section{Copyright Disclaimer}

Copyright reserved by the author(s).

This article is an open-access article distributed under the terms and conditions of the Creative Commons Attribution license (http://creativecommons.org/licenses/by/3.0/). 